\title{
Refocusing schemes for holonomic quantum computation in presence of dissipation
}

\author{
Li-Xiang Cen and Paolo Zanardi \\ Institute for Scientific Interchange Foundation, Viale Settimio Severo 65, I-10133 Torino, Italy
}

\begin{abstract}
The effects of dissipation on a holonomic quantum computation scheme are analyzed within the quantum-jump approach. We extend to the non-Abelian case the refocusing strategies formerly introduced for (Abelian) geometric computation. We show how double loop symmetrization schemes allow one to get rid of the unwanted influence of dissipation in the no-jump trajectory.
\end{abstract}

PACS numbers: 03.67.Lx, 03.65.Vf, 03.65.Yz

Due to the fragility of quantum coherence in the presence of noise, decoherence is the main obstacle to the practical realization of quantum computation. Exploring potential ways to implement robust quantum computation therefore is a crucial and attractive challenge. To the aim of stabilizing quantum information a variety of decoherence-reduction techniques have been developed, such as quantum error correcting codes 1, 2, 3], decoherence-free subspaces [4, 5] and generalized decoupling techniques [6].

The geometric/holonomic approach to quantum computation is believed to provide an intriguing geometrical way to protect quantum information processing 7, 8, 9, 10, 11, 12, 13. Geometric quantum effects, either the simple Abelian phase factor 14] or its general non-Abelian counterpart 15], arise when a quantum system is adiabatically driven to undergo an appropriate cyclic evolution. A purely geometric transformation can be obtained via a refocusing scheme [8] which cancels out the dynamical phase or by properly designing the system levels [7, 10] to avoid the dynamical phase directly.

The robustness of geometric quantum computation has been intensively addressed recently 16, 17, 18, 19, 20, 21]. For a classical noise, the inherent stability of Berry phase has been clearly demonstrated by studying a spin one-half system subject to a randomly fluctuating magnetic field [18].

In this paper we will consider geometric manipulations, Abelian and non Abelian, in presence of quantum dissipation. We will first analyze, the quantum-jump formalism, the non-unitary effects induced by dissipation on the geometrical gates in order to devise strategies to get rid of them. We will show that the geometric gate operation based on a double-loop refocusing scheme does possess a certain resilience against dissipation induced distortion. The main contribution of this paper will consist of a novel scheme which further extends the refocusing technique to enact non-Abelian holonomic quantum gates by means of a sort of simultaneous double-loop control process.

Influence of dissipation on geometric quantum computation. Let us consider the dissipative dynamics of a time-dependent periodic system governed by the following master equation

$$
\dot{\rho}=-i[H(t), \rho]-\frac{1}{2} \sum_{k=1}^{n}\left(\Gamma_{k}^{\dagger} \Gamma_{k} \rho+\rho \Gamma_{k}^{\dagger} \Gamma_{k}-2 \Gamma_{k} \rho \Gamma_{k}^{\dagger}\right) .
$$

In the framework of quantum jump approach [22], the operator $\Gamma_{k}$ is associated with jump in the trajectory; the time evolution of the system is calculated by averaging over all the possible trajectories. Stochastic jumps and diffusion of trajectories will destroy quantum coherence. This undesired effect becomes particularly severe for slow adiabatic evolutions since decoherence has a long time to bite in the process.

As one could intuitively anticipate, the geometric contributions in the evolution will show dissipation-related aspects distinct from the dynamical evolution ones. A promising approach to address this issue is to foucs on the evolution of single trajectories. In this way one can resort to the theory of geometric phases developed for pure states. Individual trajectories can be realized conditionally to the occurrence of a suitable chain of detections. Notice that this approach has been recently employed to calculate the geometric phase of open systems [19, 20].

A single trajectory in the quantum jump model is specified, for a continuously measured system, as a chain of states obtained by the action of a specific sequence of detected jumps. The state evolution conditioned to the detection of no jumps, i.e., the no-jump trajectory, can be described by the Schrödinger equation

$$
i \frac{\partial}{\partial t}|\psi(t)\rangle=\tilde{H}(t)|\psi(t)\rangle,
$$

with the non-Hermitian effective Hamiltonian given by

$$
\tilde{H}(t)=H(t)-\frac{i}{2} \sum_{k=1}^{n} \Gamma_{k}^{\dagger} \Gamma_{k}
$$

Here, the last term contained in Eq. (3) accounts for the dissipative interaction. The state evolution indicated above therefore differs from the conventional unitary dissipation-free process. This deviation then results in an inevitable distortion for the desired gate operations.

Let us first examine the Berry phase shift achieved through the double-loop refocusing scheme e.g., in an NMR system [8]. A spin half nucleus is assumed to be subjected to a cyclic field evolving adiabatically with cone angle $\theta$ and its Hamiltonian is written as $H_{0}^{C}(t)=$ $\frac{\Omega}{2}\left(\cos \theta \sigma_{z}+\sin \theta\left(\cos \gamma t \sigma_{x}+\sin \gamma t \sigma_{y}\right)\right)$. The dynamical evolution will generate a dynamical phase $\phi_{ \pm}^{d}= \pm \frac{1}{2} \Omega T$ as well as a geometric Berry phase $\phi_{ \pm}^{g}= \pm \pi(1-\cos \theta)$. To remove the dynamical phase, the cyclic process is run 
twice; the second loop is surrounded by a pair of hard $\pi$ pulses and it is driven in the opposite orientation of the first one. A pure geometric phase shift is then obtained. We consider now the system subjected to dissipation with $\Gamma=\sqrt{\kappa} \sigma_{-}$. The conditional no-jump evolution of the system is governed by the Schrödinger equation with an effective Hamiltonian (3). Instead of a real dynamical phase originated from the unitary system, dissipation will give rise to a complex "dynamical phase" given by

$\tilde{\phi}_{ \pm}^{d}= \pm \frac{1}{2} \bar{\Omega} T-\frac{i}{4} \kappa T, \quad \bar{\Omega}=\Omega\left[\sin ^{2} \theta+\left(\cos \theta-\frac{i}{2} \frac{\kappa}{\Omega}\right)^{2}\right]^{1 / 2}$.

The imaginary part of $\tilde{\phi}_{ \pm}^{d}$ describes the decay of the trajectory probability, a different decay rate is obtained for different basis states. This latter fact implies a distortion of the evolution respect to the dissipation-free process.

More specifically for the desired geometric action, equation (4) reveals that the dissipation-induced distortion compared with $\phi^{g}$ is in an order of $\kappa / \gamma$, which for an adiabatic process with small $\gamma$, can be rather severe. Nevertheless this distortion can be avoided in the whole procedure since, by removing the dynamical phase, the refocusing evolution with the opposite direction will remove the inhomogeneity of dissipation as well. The overall process gives merely origin to a global suppression of the trajectory probability with $\Gamma_{d}=e^{-\frac{1}{2} \kappa T}$.

The cancellation of the unwanted non-unitary effects achieved by the double loop technique is analog to a sort of noise decoupling by symmetrization via $\sigma_{x}$-pulses $[\underline{6}$. Indeed the kinematical symmetry $\tilde{H}_{0}^{\bar{C}}(t)=\tilde{H}_{0}^{C}(T-t)$ of the control process, along with the exchange of the computational basis states over the second loop leads to a homogeneous, i.e., global action of the dissipation that can then be neglected.

It should be noted that the effect of dissipation on the dynamical phase discussed above is not the only influence that dissipation has on the quantum dynamics of the system. Indeed, the non-unitary character of the evolution will affect the geometrical phase too. One is then lead to consider a complex Berry phase describing the dissipative effects embedded inside the geometric action,

$$
\tilde{\phi}_{ \pm}^{g}= \pm \pi\left[1-(\Omega / \bar{\Omega})\left(\cos \theta-\frac{i}{2} \frac{\kappa}{\Omega}\right)\right] .
$$

In view of the sensitivity of the geometric phase under the reversal of the orientation of the second control loop the dissipative effects in Eq. (5) cannot be removed via the refocusing process. On the other hand, in case of low dissipation, equation (5) reveals that the resulting error is of order $\kappa / \Omega$ and thus can be made negligible by making $\Omega$ large enough.

Dissipation in holonomic quantum computation. We turn now to consider another well-established approach, namely, the so-called holonomic quantum computation (HQC) 7]. In HQC information is encoded in a degenerate eigenspace of the governing Hamiltonian. Pure geometric operations, including the Abelian phase factor and non-Abelian transformation, are naturally achieved by adiabatic evolutions. Dynamical phase induced herein is clearly global due to the inherent structural symmetry (degeneracy) of the system levels. Dissipation occurring in this system can be divided into two categories: (i) dissipation preserving the degeneracy structure and (ii) inhomogeneous dissipation lifting the degeneracy. It can be easily seen that in case (i) of homogeneous dissipation, only global decay can be induced. Hence the distortion to the evolution can be avoided in the no-jump trajectory [23. In what follows we shall focus on the general case of inhomogeneous dissipation, which will affect the cyclic evolution of the system in a more severe manner.

Consider as an illustration the standard optical system 10, 12 in which a qubit is encoded in a multi-level $\Lambda$ type trapped ion or a similar cavity atom. The ground (or metastable) levels $\left|g_{i}\right\rangle(i=1, \cdots, n)$ are highly degenerated and each couples to the excited state $|e\rangle$ in a tunable manner. Let the states $\left|g_{1}\right\rangle$ and $\left|g_{2}\right\rangle$ correspond to the computational basis $|0\rangle$ and $|1\rangle$, respectively. In order to obtain the gate operation $e^{i \phi^{g}|1\rangle\langle 1|}$, the system is described by the periodic Hamiltonian

$$
H_{C}(\theta, \varphi)=\Omega \sin \theta\left(\sigma_{2 e}+\sigma_{e 2}\right)+\Omega \cos \theta\left(\sigma_{3 e} e^{i \varphi}+\sigma_{e 3} e^{-i \varphi}\right),
$$

where $\sigma_{e i}:=|e\rangle\left\langle g_{i}\right|(i=1,2,3)$, and the parameters $\theta, \varphi$ evolve adiabatically. In the dissipation-free case the dark state of the system given by $|D(\theta, \varphi)\rangle=$ $\cos \theta\left|g_{2}\right\rangle-\sin \theta e^{i \varphi}\left|g_{3}\right\rangle$, after a loop in the parameter space acquires a net Berry phase $\phi^{g}$ which depends only on the solid angle of the loop. Suppose now that the level $\left|g_{3}\right\rangle$ is metastable and suffers damping with $\Gamma^{\dagger} \Gamma=\kappa_{3} \sigma_{33}$. One has that $\left\langle D(\theta, \varphi)\left|\kappa_{3} \sigma_{33}\right| D(\theta, \varphi)\right\rangle=\kappa_{3} \sin ^{2} \theta$. The dissipative dynamics lifts the degeneracy between the dark state and the ground state $\left|g_{1}\right\rangle$. The evolution of the nojump trajectory is described by the following non-unitary transformation

$$
|\psi(t)\rangle=\frac{u_{C}|\psi(0)\rangle}{\sqrt{\left\langle\psi(0)\left|u_{C}^{\dagger} u_{C}\right| \psi(0)\right\rangle}},
$$

where the operator $u_{C}$ is given by

$$
u_{C} \simeq e^{\left(\tilde{\phi}^{d}+i \phi^{g}\right)|1\rangle\langle 1|},
$$

with $\tilde{\phi}^{d}=-\frac{1}{2} \int_{0}^{T} \kappa_{3} \sin ^{2} \theta d t$. In detail, for the typical process in which $\varphi=\gamma t$ rotates uniformly, one has explicitly $\phi^{g}=4 \pi \sin ^{2} \theta$ and $\tilde{\phi}^{d}=-\pi\left(\kappa_{3} / \gamma\right) \sin ^{2} \theta$. Dissipation induced distortion in this process scales as $\kappa_{3} / \gamma$, which turns out to be unfavorable since for the adiabatic processing, the damping rate $\kappa_{3}$ may be comparable to the parameter frequency $\gamma$. Note also that in the above calculation, a low damping rate has been assumed: we have ignored the influence of dissipation on the geometric phase $\phi^{g}$ and the deviation to the instantaneous dark state. Both of these effects would cause an error to the gate operation scaling as $\kappa_{3} / \Omega$. This latter ratio we assume can be made negligible. 
Let us investigate further the influence of dissipation on the other single-qubit holonomic gate studied in [10]. We consider the adiabatic evolution generated by the Hamiltonian

$$
\begin{aligned}
H(t)= & \Omega \sin \theta\left[\cos \varphi\left(\sigma_{1 e}+\sigma_{e 1}\right)+\sin \varphi\left(\sigma_{2 e}+\sigma_{e 2}\right)\right] \\
& +\Omega \cos \theta\left(\sigma_{3 e}+\sigma_{e 3}\right) .
\end{aligned}
$$

The system admits two dark states $\left|D_{1}\right\rangle=$ $\cos \theta\left(\cos \varphi\left|g_{1}\right\rangle+\sin \varphi\left|g_{2}\right\rangle\right)-\sin \theta\left|g_{3}\right\rangle$ and $\left|D_{2}\right\rangle=$ $\cos \varphi\left|g_{2}\right\rangle-\sin \varphi\left|g_{1}\right\rangle$. A suitable cyclic evolution in the dissipation-free process will generate the non-Abelian holonomic gate operation $e^{i \phi^{g} \sigma_{y}}$; the parameter $\phi^{g}$ describes again the solid angle swept by the executed loop. We assume the same dissipation of the metastable level $\left|g_{3}\right\rangle$. It should be noted that the dissipation generated dynamics for the two dark states does not commute with the non-Abelian connection. In view that $\left\langle D_{i}\left|\kappa_{3} \sigma_{33}\right| D_{j}\right\rangle=\kappa_{3} \sin ^{2} \theta$ for $i=j=1$ and null for other choices of $i$ and $j$, a perturbation calculation gives directly that

$$
u_{C} \simeq e^{\tilde{\phi}^{d}}|0\rangle\langle 0|+i \phi^{g} \sigma_{y},
$$

where $\tilde{\phi}^{d}=-\frac{1}{2} \int_{0}^{T} \kappa_{3} \sin ^{2} \theta d t$. For the specified process with $\varphi=\gamma t$, one has $\phi^{g}=2 \pi \cos \theta$ and $\tilde{\phi}^{d}=$ $-\pi\left(\kappa_{3} / \gamma\right) \sin ^{2} \theta$. Similar to the former case, the distortion induced by inhomogeneous dissipation to this gate is also of order $\kappa_{3} / \gamma$.

Generalized refocusing scheme for holonomic quantum computation. We have shown in the above the influence of dissipation on two different schemes for geometric quantum computation. At this stage, an interesting question arises: Is the refocusing strategy described in the former scheme extendable to the case of non-Abelian holonomic operations? In what follows we will explicitly address that question for the kind of optical system proposed in [10]. The answer will turn out to be "yes".

It is worthwhile to stress the role of symmetry in the dynamical evolutions of above systems. The kinematical symmetry of refocusing scheme, i.e., $H_{c}(t)=H_{\bar{c}}(T-t)$, helps in quenching the dissipation-induced distortion. This is due to the fact that refocusing removes the dynamical contributions from the evolution and these latter are the ones which are more severely affected by dissipation. On the other hand, the symmetry associated with the level degeneracy in the holonomic scheme is lifted by the dissipative interaction. This in turn gives rise to an inhomogeneous decay of basis states and to a corresponding distortion of the holonomic gates. The scheme we are going to discuss in the following basically combines the kinematical symmetry of the double-loop refocusing scheme with a technique for restoring the dark-state symmetry and therefore homogeneity of the decay process.

In brief the novel refocusing scheme for holonomic quantum computation that we are going to analyze, can be described as a double-loop configuration in which the additional loop refocuses the first one in an oppo- site direction. As one may see, the opposite loop execution would induce the inverse operation hence cancel the desired transformation, but this can be side stepped through interchanging the roles of the two computational bases in the twice loop evolutions.

Let us start by considering in a detailed fashion the single-qubit gate $e^{i \phi^{g}|1\rangle\langle 1|}$; in the new scheme this gate can be achieved simply by performing the loop evolution twice, the first one as in (6) followed by a second one described by

$H_{\bar{C}}(\theta, \varphi)=\Omega \sin \theta\left(\sigma_{1 e}+\sigma_{e 1}\right)+\Omega \cos \theta\left(\sigma_{3 e} e^{i \varphi}+\sigma_{e 3} e^{-i \varphi}\right)$,

where the parameters $\theta$ and $\varphi$ refocus the first loop but in the opposite direction. Note in the evolution of Eq. (111) the level $\left|g_{1}\right\rangle$ was employed to replace $\left|g_{2}\right\rangle$ in the first loop of Eq. (6). We have interchanged the roles of the two basis states $\left|g_{1}\right\rangle$ and $\left|g_{2}\right\rangle$ in order to make the overall dynamical evolution symmetric with respect the exchange of states of the computational basis. This sort of symmetrization plays the role of the two hard $\pi$ pulses used in the former NMR scheme to flip the basis states.

The dissipative dynamics on the second loop (11) will generate the transformation with

$$
u_{\bar{C}} \simeq e^{\left(\tilde{\phi}^{d}-i \phi^{g}\right)|0\rangle\langle 0|} .
$$

Thus the total two-loop process will be described by the following overall transformation

$$
\begin{aligned}
u_{d l} & \simeq e^{\left(\tilde{\phi}^{d}-i \phi^{g}\right)|0\rangle\langle 0|} \times e^{\left(\tilde{\phi}^{d}+i \phi^{g}\right)|1\rangle\langle 1|} \\
& =e^{\tilde{\phi}^{d}} e^{-i \phi^{g}} e^{i 2 \phi^{g}|1\rangle\langle 1|},
\end{aligned}
$$

where $e^{-i \phi^{g}}$ denotes an irrelevant global phase. From this equation it can be clearly seen, in view of the symmetric role played by $|0\rangle$ and $|1\rangle$, that dissipation gives rise just to a global factor $e^{\tilde{\phi}^{d}}$ affecting just the no-jump trajectory probability; the former distortion in equation (8) has been suppressed.

We now discuss the extension of the refocusing strategy to the second single-qubit gate considered in [10], i.e., $e^{i \phi^{g} \sigma_{y}}$; this extension turns out to be more difficult than the previous one. Indeed, one may naively expect that the refocusing loop is described by

$$
\begin{aligned}
H_{\bar{C}}(\theta, \varphi)= & \Omega \sin \theta\left[\cos \varphi\left(\sigma_{2 e}+\sigma_{e 2}\right)+\sin \varphi\left(\sigma_{1 e}+\sigma_{e 1}\right)\right] \\
& +\Omega \cos \theta\left(\sigma_{3 e}+\sigma_{e 3}\right)
\end{aligned}
$$

with the parameter $\theta$ and $\varphi$ evolving in the opposite direction of the first loop (9). However, it is immediate to verify that the evolution associated to the loop (14), namely the transformation $u_{\bar{C}}=e^{\tilde{\phi}^{d}|1\rangle\langle 1|+i \phi^{g} \sigma_{y}}$, does not commute with that of Eq. (10). In other terms the noncommutativity of the two loop transformations destroys the desired symmetry aimed by the refocusing process. As a result such a double-loop evolution does not offer any clear advantage as far as resilience against dissipation is concerned. One can overcome this obstacle 
by performing the two opposite controlled evolutions in sense simultaneously. As the superposed process shall lead overlapped interactions to the level $\left|g_{3}\right\rangle$, an extra level will be necessary to pin it down. Explicitly, the whole process of the dual-superposed loop evolution can be described by the following periodic Hamiltonian

$$
\begin{aligned}
H_{d l}= & \Omega \sin \theta\left[(\cos \varphi-\sin \varphi) \sigma_{1 e}+(\sin \varphi+\cos \varphi) \sigma_{2 e}\right] \\
& +\Omega \cos \theta\left(\sigma_{3 e}+\sigma_{4 e}\right)+h . c .
\end{aligned}
$$

Here the level $\left|g_{3}\right\rangle$ and the extra level $\left|g_{4}\right\rangle$ are supposed to suffer the homogeneous damping $\Gamma^{\dagger} \Gamma=\kappa_{3}\left(\sigma_{33}+\sigma_{44}\right)$. As the parameters $\theta$ and $\varphi$ evolve adiabatically, the two dark states of the newly established Hamiltonian (15),

$$
\begin{aligned}
\left|D_{1}^{d l}\right\rangle & =\cos \theta\left(\cos \varphi\left|g_{1}\right\rangle+\sin \varphi\left|g_{2}\right\rangle\right)-\sin \theta\left|g_{3}\right\rangle \\
\left|D_{2}^{d l}\right\rangle & =\cos \theta\left(\cos \varphi\left|g_{2}\right\rangle-\sin \varphi\left|g_{1}\right\rangle\right)-\sin \theta\left|g_{4}\right\rangle
\end{aligned}
$$

shall generate a non-Abelian holonomy and it can implement the identical gate operation $e^{i \phi^{g} \sigma_{y}}$ with $\phi^{g}$ denoting the loop-related solid angle. The advantage of the present system is obvious: the yielded new dark states possess completely symmetric structure which is thus capable to remove the inhomogeneous effect of dissipation on the computational space. Simply the dissipation can merely lead to a global decay and for the no-jump trajectory the transformation is described by

$$
u_{d l} \simeq e^{\tilde{\phi}^{d}} e^{i \phi^{g} \sigma_{y}}
$$

It should be pointed out that the loop superposition strategy is also realizable for the previously discussed single-qubit holonomic gate, which thus provides an alternative way to implement the gate $e^{i \phi^{g}|1\rangle\langle 1|}$. We stress here that the homogeneity achieved in the loopsuperposed processes is restricted on the two-dimensional subspace spanned by the computational basis.

In this paper we analyzed the robustness of geometric quantum gates, both Abelian and non-Abelian, against quantum dissipation. We have adopted the quantum jump approach to describe dissipative dynamics and we focused on the no-jump trajectory. It has been shown that geometric gates realized by means of a refocused double-loop scheme possess a certain resilience against dissipation-induced distortion. This robustness is due to the underlying symmetry of the scheme with respect to the exchange of computational basis states. The crucial point is that the non-unitarity affects mostly the dynamical phases; these latter can be removed by a suitable control process which leaves the geometrical part of the evolution untouched. The power of this refocusing strategy has been demonstrated by extending it to non-Abelian holonomic gates. For the non-Abelian case, we have shown how the same sort of symmetrization can be achieved via a loop superposition strategy. We conclude by noticing the central role of symmetry in a variety of schemes of quantum information processing (see e.g., Ref. [24] and references therein). The present work reveals one more intriguing way in which symmetry might help in our struggle against decoherence.

This work is financially supported by the European Union project TOPQIP (Contract No. IST-2001-39215).
[1] P.W. Shor, Phys. Rev. A 52, 2493 (1995); A.M Steane, Phys. Rev. Lett. 77, 793 (1997).

[2] E. Knill and R. Laflamme, Phys. Rev. A 55, 900 (1997).

[3] D. Gottesman, Phys. Rev. A 54, 1862 (1996).

[4] P. Zanardi and M. Rasetti, Phys. Rev. Lett. 79, 3306 (1997); Mod. Phys. Lett. B 11, 1085 (1997).

[5] D.A. Lidar, I.L. Chuang, and K.B. Whaley, Phys. Rev. Lett. 81, 2594 (1998).

[6] L. Viola and S. Lloyd, Phys. Rev. A 58, 2733 (1998); L. Viola, E. Knill, and S. Lloyd, Phys. Rev. Lett. 82, 2417 (1999); P. Zanardi, Phys. Lett. A 258, 77 (1999).

[7] P. Zanardi and M. Rasetti, Phys. Lett. A 264, 94 (1999).

[8] J.A. Jones, V. Vedral, A. Ekert and G. Castagnoli, Nature (London) 403, 869 (2000).

[9] G. Falci, R. Fazio, G.M. Palma, J. Siewert and V. Vedral, Nature (London) 407, 355 (2000).

[10] L.M. Duan, J.I. Cirac and P. Zoller, Science 292, 1695 (2001).

[11] J. Pachos, P. Zanardi, and M. Rasetti, Phys. Rev. A 61, 010305(R) (2002).

[12] A. Recati, T. Calarco, P. Zanardi, J.I. Cirac and P. Zoller, Phys. Rev. A 66, 0302309 (2002).

[13] L.X. Cen, X.Q. Li, Y.J. Yan, H.Z. Zheng, and S.J. Wang,
Phys. Rev. Lett. 90, 147902 (2003).

[14] M.V. Berry, Proc. R. Soc. London, Ser. A 392, 45 (1984); B. Simon, Phys. Rev. Lett. 51, 2167 (1983).

[15] F. Wilczek and A. Zee, Phys. Rev. Lett. 52, 2111 (1984).

[16] A. Nazir, T.P. Spiller, and W.J. Munro, Phys. Rev. A 65, 042303 (2002).

[17] A. Blais and A.-M. S. Tremblay, Phys. Rev. A 67, 012308 (2003).

[18] G. DeChiara and G.M. Palma, Phys. Rev. Lett. 91, 090404 (2003).

[19] A. Carollo, I. Fuentes-Guridi, M.F. Santos, and V. Vedral, Phys. Rev. Lett. 90, 160402 (2003).

[20] A. Carollo, I. Fuentes-Guridi, M.F. Santos, and V. Vedral, Phys. Rev. Lett. 92, 020402 (2004).

[21] I. Fuentes-Guridi, F. Girelli, and E. Livine, quant-ph/0311164

[22] M.B. Plenio and P.L. Knight, Rev. Mod. Phys. 70, 101 (1998).

[23] In case of homogeneous dissipation, the trajectories with jumps occurring have also been discussed in Ref. [21].

[24] P. Zanardi, and S. Lloyd, Phys. Rev. Lett. 90, 067902 (2003). 